\title{
MOST SIMILARITY ORBITS ARE STRONGLY DENSE ${ }^{1}$
}

\author{
D. W. HADWIN, E. A. NORDGREN, HEYDAR RADJAVI AND \\ PETER ROSENTHAL
}

\begin{abstract}
It is shown that the strong and weak closures of the similarity orbit of an operator on a Banach space always coincide, and a simple characterization of these closures is given. Whenever an operator is not the sum of a scalar and a finite rank operator, its similarity orbit is strongly dense in the set of all bounded linear operators.
\end{abstract}

Characterizing the norm closure of the similarity orbit $\delta(T)=\left\{A^{-1} T A: A\right.$ is invertible \} of a bounded linear operator $T$ on a Banach space is a very difficult task (supporting evidence is contained in the work of D. A. Herrero [He1], [He2]). The norm closure of the unitary equivalence class of an operator on a Hilbert space is more tractable, but the characterization due to D. Voiculescu [V] (see also [H1], [H2]) is very deep and complicated.

One way to make the problem easier is by taking closures in more manageable operator topologies (i.e., strong, weak) than the norm topology. The weak and strong closures of the unitary equivalence class $\mathcal{U}(T)$ of an operator $T$ on a Hilbert space are usually not the same, and they are generally more difficult to describe than the norm closure [H2] (e.g., if $T$ is normal with $\sigma(T)$ equal to the closed unit disk, then the norm closure of $\mathcal{U}(T)$ is the set of all normal operators $S$ with $\sigma(S)=\sigma(T)$, the strong closure of $\mathcal{U ( T )}$ is the set of all subnormal operators $S$ with $\|S\|<1$, and the weak closure of $\mathscr{U}(T)$ is the set of all operators $S$ with $\|S\|<1)$.

In this note we show that the weak and strong closures of the similarity orbit of an operator on an infinite-dimensional Banach space $X$ always coincide, and they are almost always equal to $B(X)$, the set of all operators on $X$.

THEOREM 1. If $T \in B(X)$ and $T$ is not the sum of a scalar and a finite rank operator, then $\mathcal{S}(T)$ is strongly (thus, weakly) dense in $B(X)$.

TheOReM 2. If $T \in B(X)$ and $\operatorname{rank}(T-\lambda)=m<\infty$ for some scalar $\lambda$, then the strong (weak) closure of $\delta(T)$ is $\{\lambda+F$ : rank $F<m\}$.

The proofs of the theorems are based on the following facts:

I. If $n$ is a positive integer, then $\{S \in B(X)$ : rank $S<n\}$ is weakly closed.

Received by the editors September 5, 1978 and, in revised form, November 28, 1978.

AMS (MOS) subject classifications (1970). Primary 47A99.

Key words and phrases. Banach space, operator, similarity orbit, weak and strong operator topologies, finite rank operator.

${ }^{1}$ Supported in part by a grant from the National Science Foundation. 
II. If $T \in B(X)$ and $T$ is not the sum of a scalar and a finite rank operator, then, for each positive integer $n$, there are vectors $g_{1}, g_{2}, \ldots, g_{n}$ in $X$ such that $\left\{g_{1}, g_{2}, \ldots, g_{n}, T g_{1}, T g_{2}, \ldots, T g_{n}\right\}$ is linearly independent.

Statement I can be proved by a slight modification of the proof of P. R. Halmos [PRH1] for the Hilbert space case. To prove statement II assume that $\left\{g_{1}, g_{2}, \ldots, g_{n}\right\}$ is maximal with respect to the property that $\left\{g_{1}, \ldots, g_{n}\right.$, $\left.T g_{1}, \ldots, T g_{n}\right\}$ is linearly independent. Choose an idempotent $P$ in $B(X)$ whose range is the linear span of $\left\{g_{1}, \ldots, g_{n}, T g_{1}, \ldots, T g_{n}\right\}$. It follows from maximaility that every vector in the kernel of $P$ is an eigenvector for $(1-P) T(1-P)$. Thus

$$
(1-P) T(1-P)=\lambda(1-P)
$$

for some scalar $\lambda$. Hence $\operatorname{rank}(T-\lambda)<\infty$.

Proof of Theorem 1. Suppose $S \in B(X)$ and $V$ is a strong neighborhood of $S$. Then there are linearly independent vectors $e_{1}, \ldots, e_{n}$ in $X$ and a positive number $\varepsilon$ such that $V$ contains $\left\{A \in B(X):\left\|(A-S) e_{k}\right\|<\varepsilon\right.$ for $1<k<n\}$. Since $X$ is infinite-dimensional, we can choose vectors $f_{1}, \ldots, f_{n}$ in $X$ so that $\left\{e_{1}, e_{2}, \ldots, e_{n}, f_{1}, f_{2}, \ldots, f_{n}\right\}$ is linearly independent and $\left\|S e_{k}-f_{k}\right\|<\varepsilon$ for $1 \leqslant k \leqslant n$. By statement II, there are vectors $g_{1}, g_{2}, \ldots, g_{n}$ in $X$ such that $\left\{g_{1}, \ldots, g_{n}, T g_{1}, \ldots, T g_{n}\right\}$ is linearly independent. If $A$ is any invertible operator such that $A e_{k}=g_{k}$ and $A f_{k}=T g_{k}$ for $1<k<n$, then $\left\|\left(A^{-1} T A-S\right) e_{k}\right\|<\varepsilon$ for $1 \leqslant k \leqslant n$. Thus $A^{-1} T A \in V$.

Proof of TheOrem 2. Clearly, we can assume that $\lambda=0$. Statement I says that $\{F \in B(X)$ : rank $F \leqslant m\}$ is weakly closed; hence, it suffices to show that this set coincides with the strong closure of $\delta(T)$. Suppose $k=\operatorname{rank} F$ $<m$, and, following the preceding proof, suppose $\varepsilon>0$ and $\left\{e_{1}, \ldots, e_{n}\right\}$ is linearly independent. Since rank $F<\infty$, the set $\left\{e_{1}, e_{2}, \ldots, e_{n}\right\}$ is contained in a finite-dimensional invariant subspace of $F$. Hence we can assume that $F e_{i}=0$ for $k<i \leqslant n$. (Otherwise, replace $e_{1}, \ldots, e_{n}$ by a basis for this invariant subspace and $\varepsilon$ by an appropriately smaller positive number.) Choose vectors $f_{1}, \ldots, f_{k}$ in $X$ so that $\left\{e_{1}, \ldots, e_{n}, f_{1}, \ldots, f_{k}\right\}$ is linearly independent and $\left\|F e_{i}-f_{i}\right\|<\varepsilon$ for $1 \leqslant i \leqslant k$. Since $k \leqslant \operatorname{rank} T<\infty$ and $X$ is infinite-dimensional, we can choose vectors $g_{1}, \ldots, g_{n}$ in $X$ so that $\left\{g_{1}, \ldots, g_{n}, T g_{1}, \ldots, T g_{k}\right\}$ is linearly independent and $T g_{i}=0$ for $k<i<$ $n$. If $A$ is any invertible operator such that $A e_{i}=g_{i}$ for $1<i<n$ and $A f_{j}=T g_{j}$ for $1 \leqslant j \leqslant k$, then $\left\|\left(A^{-1} T A-F\right) e_{j}\right\|<\varepsilon$ for $1<j<n$.

Remarks. 1 . Note that Theorems 1 and 2 can be combined into the simple (but less informative) statement: the strong (weak) closure of $\delta(T)$ is $\{S$ : $\operatorname{rank}(S-\lambda) \leqslant \operatorname{rank}(T-\lambda)$ for every scalar $\lambda\}$.

2. One useful type of application of Theorem 1 is in showing that certain classes of operators are strongly dense. Here are some examples:

(a) It was shown by P. R. Halmos [PRH2], using an idea of A. Lebow which is similar to the proof of Theorem 1 above, that the set of operators $T$ with $T^{2}=0$ is strongly dense in $B(X)$. This result is an immediate con- 
sequence of Theorem 1 . In fact, a more general result is true. If $p(z)$ is a polynomial with degree greater than 1 , then $\{T \in B(X): p(T)=0\}$ is strongly dense in $B(X)$.

(b) In [HNRR] the authors constructed an operator $T$ on a Hilbert space $H$ whose commutant contains no nonscalar operator that commutes with a nonzero compact operator. It follows from Theorem 1 that the set of all such operators is strongly dense in $B(H)$.

(c) A transitive operator on $X$ is an operator whose only invariant subspaces are $\{0\}$ and $X$. It follows from Theorem 1 that the set of transitive operators on $X$ is either empty or strongly dense in $B(X)$. (See [E].)

3. When $X$ is a Hilbert space, there is another useful topology on $B(X)$, the *-strong operator topology. What is the *-strong closure of $\delta(T)$ for $T \in$ $B(X)$ ?

4. Suppose $X$ is a separable, infinite-dimensional Hilbert space. It is possible to show that if $T$ is not the sum of a scalar and a finite rank operator, then $\delta(T)$ is sequentially weakly dense in $B(X)$. Here "weak" cannot be replaced by "strong" (e.g., consider the algebraic operators). The proof of this fact makes use of deep results in [Hel] and [H2]. Is there a simpler proof of this fact?

\section{REFERENCES}

[E] P. Enflo, On the invariant subspace problem in Banach spaces, Séminaire Maurey-Schwartz 1975-1976: Espaces $L^{p}$, applications radonifiantes et géométrie des espaces de Banach, Exposés Nos. 14-15, Centre Math., Ecole Polytech., Palaiseau, 1976, 6 pp.

[H1] D. W. Hadwin, An operator-valued spectrum, Indiana Univ. Math. J. 26 (1978), 329-340.

[H2] __ Approximate equivalence and completely positive maps, preprint, 1978.

[HIRR] D. W. Hadwin, E. Nordgren, Heydar Radjavi and Peter Rosenthal, An operator not satisfying Lomonosov's hypothesis, preprint, 1978.

[PRH1] P. R. Halmos, Irreducible operators, Michigan Math. J. 15 (1968), 215-223.

[PRH2] __ A Hilbert space problem book, Van Nostrand, Princeton, N. J., 1967.

[He1] D. A. Herrero, Closure of similarity orbits of Hilbert space operators. III, Math. Ann. 232 (1978), 195-204.

[He2] _ Closure of similarity orbits of Hilbert space operators. V. Essentially BQT operators (preprint).

[V] D. Voiculescu, A non-commutative Weyl-von Neumann theorem, Rev. Roumaine Math. Pures Appl. 21 (1976), 97-113.

Departiment of Mathematics, University of New Hampshire, Durham, New Hampshire 03824 (Current address of D. W. Hadwin and E. A. Nordgren)

Department of Mathematics, Dalhousie University, halifax, Nova Scotia, Canada (Current address of Heydar Radjavi)

Department of Mathematics, University of Toronto, Toronto, Ontario, Canada (Current address of Peter Rosenthal) 